\title{
Whole Earth Telescope observations of the pulsating hot white dwarf PG 1707+427
}

\author{
S. D. Kawaler ${ }^{1}$, E. M. Potter ${ }^{1}$, M. Vučković ${ }^{1}$, Z. E. Dind ${ }^{2}$, S. O’Toole ${ }^{2,3}$, J. C. Clemens ${ }^{4}$, M. S. O’Brien ${ }^{5}$, \\ A. D. Grauer ${ }^{6}$, R. E. Nather ${ }^{7}$, P. A. Moskalik ${ }^{8}$, C. F. Claver ${ }^{9}$, G. Fontaine ${ }^{10}$, F. Wesemael ${ }^{10}$, P. Bergeron ${ }^{10}$, \\ G. Vauclair ${ }^{11}$, N. Dolez ${ }^{11}$, M. Chevreton ${ }^{12}$, S. J. Kleinman ${ }^{13}$, T. K. Watson ${ }^{14}$, M. A. Barstow ${ }^{15,16}$, \\ A. E. Sansom ${ }^{16,17}$, D. E. Winget ${ }^{7}$, S. O. Kepler ${ }^{18}$, A. $\operatorname{Kanaan}^{19}$, P. A. Bradley ${ }^{20}$, \\ J. Dixson ${ }^{7}$, J. Provencal ${ }^{21}$, and T. R. Bedding ${ }^{2}$ \\ ${ }^{1}$ Department of Physics and Astronomy, Iowa State University, Ames, IA 50011, USA \\ e-mail: sdk@iastate.edu \\ 2 School of Physics, University of Sydney, Sydney NSW, Australia \\ 3 Dr. Remeis-Sternwarte, Astronomisches Institut der Universität Erlangen-Nürnberg, Sternwartstr. 7, Bamberg 96049, \\ Germany \\ ${ }^{4}$ Department of Physics and Astronomy, University of North Carolina, Chapel Hill, NC 27599-3255, USA \\ 5 Department of Astronomy, Yale University, New Haven, CT 06851, USA \\ ${ }^{6}$ Department of Physics and Astronomy, University of Arkansas at Little Rock, Little Rock, AR 72204, USA \\ 7 Department of Astronomy, University of Texas, Austin, TX 78712, USA \\ 8 Nicolas Copernicus Astronomical Center, Polish Academy of Sciences, ul. Bartycka 18, 00-716 Warsaw, Poland \\ 9 National Optical Astronomy Observatory, 950 N. Cherry Ave, Tucson, AZ 85719, USA \\ 10 Département de Physique, Université de Montréal, Montréal, Québec, H3C 3J7, Canada \\ 11 Université Paul Sabatier, Observatoire Midi-Pyrénées, 14 avenue E. Belin, 31400 Toulouse, France \\ 12 Observatoire de Paris-Meudon, DAEC, 92195 Meudon, France \\ 13 Apache Point Observatory, PO Box 59, Sunspot, NM 88349, USA \\ 14 Southwestern University, 1001 E. University Avenue, Georgetown, TX 78626, USA \\ 15 Department of Physics and Astronomy, University of Leicester, Leicester LE1 7RH, UK \\ 16 Isaac Newton Group of Telescopes, 37800 Santa Cruz de La Palma, Canary Islands, Spain \\ 17 Centre for Astrophysics, University of Central Lancashire, Preston, Lancashire, PR1 2HE, UK \\ 18 Instituto de Física, UFRGS, Campus do Vale, CP 15051 Porto Alegre, RS, Brazil \\ 19 Departamento de Física, Universidade Federal de Santa Catarina, CP 4796-CDP 88040-900 Florianópolis, SC-Brazil \\ ${ }^{20}$ Los Alamos National Laboratory, X-2, MS T-085, Los Alamos, NM 87545, USA \\ ${ }^{21}$ Department of Physics and Astronomy, University of Delaware, 223 Sharp Laboratory, Newark, DE 19716, USA
}

Received 15 June 2004 / Accepted 27 August 2004

\begin{abstract}
We report on the analysis of multisite time-series photometry of the pulsating pre-white dwarf (GW Vir star) PG 1707+427, obtained by the Whole Earth Telescope collaboration. This is the last of the known GW Vir stars without surrounding nebulae to be resolved by multisite data. Successful resolution of the pulsation spectrum resulted from the combination of high signal-to-noise observations with a large telescope and wide coverage in longitude with smaller telescopes. We find a series of 8 pulsation frequencies (along with two nonlinear combination frequencies), and identify 7 of them as part of a sequence of $\ell=1$ modes, with a common period spacing of $23.0 \mathrm{~s}$. This spacing implies that the mass of PG 1707+427 is $0.57 M_{\odot}$. Preliminary model fits suggest that the mass determined via asteroseismology is consistent with the mass determined from spectroscopy combined with evolutionary tracks.
\end{abstract}

Key words. stars: evolution - stars: white dwarfs - stars: oscillations - stars: individual: PG 1707+427

\section{Introduction}

The surfaces of most stars heat up relatively quickly following their departure from the asymptotic giant branch (AGB). Most of the time, such a process eventually leads to a hot, hydrogenrich white dwarf which then gradually cools along the white dwarf cooling track. Occasionally, however, the life of a star following the AGB phase can be much more interesting. In the case where a star leaves the AGB at a late phase within the helium shell thermal pulse cycle, it can undergo a thermal pulse while near the top of the white dwarf cooling track. In that case, with only about $10^{-3} M_{\odot}$ of hydrogen in its envelope, the final 
thermal pulse briefly returns the star to the AGB. During this and subsequent evolution, the remaining hydrogen is removed through mass loss and nuclear burning.

This scenario, proposed by Iben et al. (1983) and Iben (1984) and known colloquially as the "born-again" AGB phase, could be responsible for the origin of the PG 1159 spectral class of hot white dwarfs that show extreme hydrogen depletion and enhancement of atmospheric carbon and oxygen. This mechanism for producing such objects has been explored computationally by Iben \& MacDonald (1995) and others. Subsequent theoretical work revealed that other channels could be responsible for the production of PG 1159 stars (e.g. Herwig 2001; Werner 2001, and references therein). Evidence that C/O rich central stars of planetary nebulae ([WC] central stars, which could be precursors to the naked PG 1159 stars) do not follow a single evolutionary path is presented by Górny \& Tylenda (2000) and De Marco (2002). Lawlor \& MacDonald (2003) address the connection between rapidly-evolving post-AGB stars such as Sakurai's Object and the PG 1159 stars, including detailed evolutionary models through the late (and very late) thermal pulse phases.

Two avenues for further observational study may help clarify our understanding the origin of PG 1159 stars, and hydrogen-deficient central stars. First, optical and UV spectroscopy, always of utility in astrophysics, can help in determining trace element abundances that can be markers for late mixing in post-AGB stars as well as s-process nucleosynthesis tracers (Lawlor \& MacDonald 2003; Herwig 2001). A second avenue is to probe the subsurface layers of relevant stars through asteroseismology of the GW Vir stars. There are several members of the GW Virginis class of pulsating stars, including four PG 1159 stars, HS 2324+3944, and 10 pulsating PN central stars. Of these, however, only a subset show richenough pulsation spectra for analysis that might reveal subsurface composition transition zones. Of the "naked" GW Vir stars (those without nebulae), the clear champion here, though, is PG 1159-035 itself. Asteroseismic analysis of Whole Earth Telescope (WET) data on this star (Winget et al. 1991) by Kawaler \& Bradley (1994) revealed a composition transition from He:C:O to all $\mathrm{C}: \mathrm{O}$ at a depth of $\approx 2 \times 10^{-3} M_{\odot}$ below the surface. This is entirely consistent with the generic born-again scenario.

Two of the pulsating WC central stars have been studied with extensive multisite campaigns. Asteroseismic results for NGC 1501 (Bond et al. 1996) suggest a low mass for the star $\left(M \approx 0.55 M_{\odot}\right.$ ), which is the lowest mass determined for any white dwarf through asteroseismology. While a more detailed seismic model has not been published, the mode density may allow more information to be extracted from the data. On the other hand, the pulsating central star RX J2117+341 shows a rich mode structure; Vauclair et al. (2002) analyzed several WET runs, along with additional data, revealing many modes and determining the mass as $0.56 M_{\odot}$. The mass determinations for these two stars is based on the identified period spacing and uses the scaling between period spacing and mass of Kawaler \& Bradley (1994) (see O'Brien 2000 for a correction). One must keep in mind that that relation was derived for the naked GW Vir stars that are at much lower luminosities.
Their relatively low asteroseismic masses could be artifacts of a modest breakdown in the relation for high luminosity objects. Detailed asteroseismic modeling of RX J2117+341 could reveal its internal structure in sufficient detail for comparison with PG 1159-035 - such an analysis is underway.

In addition to the adiabatic constraints on the structure and properties of these stars, it is becoming clear that the driving mechanism responsible for the observed pulsations can provide a partially independent probe of the composition of the subsurface layers. Soon after the discovery of pulsating PG 1159 stars, Starrfield et al. (1983, 1984, 1985) identified the cyclic ionization of carbon and oxygen as a possible excitation mechanism. Later work (i.e. Gautschy 1997; Bradley \& Dziembowski 1996) placed constraints on the possible surface and subsurface helium and $\mathrm{C} / \mathrm{O}$ abundances based on vibrational instability arguments. More recently, Cox (2003) explored the importance of iron ionization on pulsation driving. Quirion et al. (2004) have also revisited this interesting question.

Within the realm where stellar models are available for full asteroseismic modeling, we need to obtain sufficient data on other members of the naked GW Vir pulsators. To confront evolutionary scenarios with more representative data, it is important to complete the survey of the pulsation properties of the naked GW Vir stars. Aside from PG 1707+427, significant results have been published for all others in its class. (PG 0122+200, O'Brien et al. 1998 and Vauclair et al. 2001; PG 2131+066, Kawaler et al. 1995; Reed et al. 2000). In each of those cases, the mode density is not high enough for detailed asteroseismic analysis at present.

O'Brien $(1998,2000)$ attempts to use the existing ensemble of pulsation properties to explore the evolutionary patterns of the naked GW Vir stars. The trends noted by O'Brien (1998, 2000) can be further tested by a more complete analysis of PG $1707+427$. Should the star show a distinguishing pulsation mode structure, it is also possible that we can constrain its internal composition profile. To that end, we have extracted data on PG 1707+427 from the Whole Earth Telescope archives, and present a fresh analysis of those data in this paper.

PG $1707+427$ was discovered to be a pulsator by Bond \& Grauer (1984). It is in most respects a garden-variety PG 1159 star, with $T_{\text {eff }}=85000 \mathrm{~K}$ and $\log g=7.5$ (Dreizler \& Heber 1998). It shows a dominant pair of periodicities at about $447 \mathrm{~s}$, with the harmonic of the larger pulsation, and the sum of the two largest, present in the Fourier transform. Other modes are present at lower amplitude, ranging from $900 \mathrm{~s}$ to about $300 \mathrm{~s}$. A single run at the CFHT at high signal-to-noise allowed Fontaine et al. (1991) to show that there are additional lowamplitude pulsation modes in PG $1707+427$. They published a preliminary determination of the mean period spacing as well the period spacing we find revises this upwards with more accurate period determinations for those modes. An intensive multiyear observing effort was presented by Grauer et al. (1992) in an attempt to measure $\mathrm{d} P / \mathrm{d} t$; unfortunately, they showed that the amplitudes and phases of the main pulsation modes were not stable on long (season-to-season) time scales.

Despite it being one of the faintest of the known pulsating GW Virginis stars ( $V=16.7, B=16.1)$, the WET attempted to obtain global time-series data on PG 1707+427 in May 1991. 
Past analysis of these data were frustrated by the relatively poor coverage, and the small number of telescopes with sufficiently large aperture. However, in the intervening time, our data reduction and analysis tools have matured considerably. By combining data from the WET network of modest signal-to-noise with data from the CFHT at much higher signal-to-noise obtained during the WET run, we are able to fully resolve the pulsation spectrum. This paper presents a fresh analysis of these data - we have been able to extract a significant number of independent pulsation modes from the data and to derive the mean period spacing. This new value, coupled with the luminosity and effective temperature of PG $1707+427$ follows the trends identified by O'Brien $(1998,2000)$.

The remainder of this paper is structured as follows. In Sect. 2, we outline our observations. Section 3 describes the time series analysis and period identifications. We discuss the pulsation spectrum and compare with theoretical expectations using a preliminary stellar model fit to PG $1707+427$ in Sect. 4. Section 5 presents our conclusions in terms of the evolutionary history of the hydrogen deficient hot white dwarfs.

\section{High speed photometry}

The WET run in May 1991 (Xcov6) included PG 1707+427. Another target, GD 154, shared priority with PG $1707+427$ for this WET run; for details on the GD 154 results see Pfeiffer et al. (1996). As PG $1701+427$ is a faint star, and telescope time was shared between it and GD 154, the only useful data we obtained was using telescopes with apertures in excess of $1 \mathrm{~m}$. The observing runs, dates, times, and observatories are listed in Table 1 . In all, we obtained over $132 \mathrm{~h}$ of data on PG $1707+427$, with $120 \mathrm{~h}$ during the 12 days of the main campaign (12-24 May 1991). The average night's data from a single site was $6.3 \mathrm{~h}$.

All sites used photoelectric photometers, with either 5, 10, or $20 \mathrm{~s}$ individual integrations (depending on the site and local observing conditions). Three-channel photometers (unfiltered) were used at the KPNO $1.3 \mathrm{~m}$ and the CFHT, while two-channel photometers were used at McDonald and at the INT $2.5 \mathrm{~m}$. All photometers were of similar design, as discussed in Nather et al. (1990) and Kleinman et al. (1996).

As is usual for WET observations, the raw data consist of "photon" counts for the target, and a nearby comparison star, observed through small apertures in the focal plane. For twochannel observations, the observers occasionally (and aperiodically) moved the telescope to an adjacent area of blank sky for sky count measurements. In the case of three-channel photometers, the third channel monitors the sky continuously, with cross calibration of the channels performed once during the night. Data from all sites were reduced, as described in Nather et al. (1990), by transforming the raw light curves through sky subtraction and extinction correction.

The reduced light curves show fractional intensity variation as a function of time. The light curve from the main data set is shown in Fig. 1. The relatively short runs from the CFHT, coupled with the sparse longitude coverage, limited our coverage of the $24 \mathrm{~h}$ diurnal cycle; it was not at the usual high duty cycle that we have come to expect for WET campaigns. Still,
Table 1. Observations of PG $1707+427$.

\begin{tabular}{ccccc}
\hline \hline Run & Date & Start time & Length & Telescope \\
& & $\mathrm{UT}$ & $\mathrm{h}$ & \\
\hline a294 & 1991.05 .12 & $4: 08: 00$ & 7.02 & KPNO 1.3 m \\
a295 & 1991.05 .13 & $5: 35: 00$ & 5.28 & KPNO 1.3 m \\
a296 & 1991.05 .14 & $4: 19: 00$ & 6.98 & KPNO 1.3 m \\
a297 & 1991.05 .15 & $3: 54: 00$ & 7.25 & KPNO 1.3 m \\
ren-0100 & 1991.05 .16 & $3: 45: 00$ & 6.52 & McDonald 2.1 m \\
int-0002 & 1991.05 .16 & $21: 53: 00$ & 7.59 & INT 2.5 m \\
ren-0101 & 1991.05 .17 & $3: 50: 00$ & 6.60 & McDonald 2.1 m \\
int-0003 & 1991.05 .17 & $21: 23: 30$ & 8.02 & INT 2.5 m \\
ren-0102 & 1991.05 .18 & $3: 32: 40$ & 6.87 & McDonald 2.1 m \\
int-0004 & 1991.05 .18 & $22: 00: 30$ & 7.42 & INT 2.5 m \\
ren-0103 & 1991.05 .19 & $3: 32: 50$ & 6.83 & McDonald 2.1 m \\
int-0005 & 1991.05 .19 & $21: 28: 00$ & 7.96 & INT 2.5 m \\
ren-0104 & 1991.05 .20 & $3: 20: 40$ & 7.02 & McDonald 2.1 m \\
cfh-002 & 1991.05 .20 & $9: 55: 53$ & 4.71 & CFHT 3.5 m \\
int-0006 & 1991.05 .20 & $21: 36: 30$ & 7.87 & INT 2.5 m \\
cfh-003 & 1991.05 .21 & $10: 32: 28$ & 4.04 & CFHT 3.5 m \\
ren-0106 & 1991.05 .23 & $8: 16: 40$ & 2.11 & McDonald 2.1 m \\
cfh-009 & 1991.05 .24 & $11: 53: 45$ & 2.83 & CFHT 3.5 m \\
& & & & \\
a305 & 1991.06 .15 & $4: 15: 00$ & 6.36 & KPNO 1.3 m \\
a306 & 1991.06 .16 & $3: 58: 00$ & 6.75 & KPNO 1.3 m \\
a307 & 1991.06 .17 & $4: 19: 00$ & 6.27 & KPNO 1.3 m \\
\hline & & & & \\
\hline
\end{tabular}

while the resulting 1 cycle/day aliases complicate the analysis, we show in the next section that we can now fully decode the pulsation spectrum of the star.

\section{The pulsation spectrum of PG $1707+427$}

\subsection{Direct identification of pulsation frequencies}

Using the light curves described in the previous section, we computed the Fourier transform (FT) to deduce the periodicities present in PG 1707+427. Figure 2 zooms in on the main features of the FT of PG $1707+427$, along with its window function. In this figure, we indicate the more obvious real frequencies (as distinguished from window features) present in the data. Clearly, though, the 1 cycle/day aliasing could be obscuring additional periodicities.

Identification of the peaks within the FT is clear for most of the groups shown in Fig. 2 except for the features around $1340 \mu \mathrm{Hz}$. In this region, prior investigations frequently cite peaks at $1329 \mu \mathrm{Hz}$ and $1350 \mu \mathrm{Hz}$. In the FT of the WET data, this region shows many peaks, along with a jumble of $1 \mathrm{c} / \mathrm{d}$ aliases.

Our identification of two real frequencies in this region takes advantage of a $17 \mathrm{~h}$ stretch of data from three sites (runs int-0005, ren-0104, and cfh-002 in Table 1). The length of this run provides a nominal frequency resolution of $16 \mu \mathrm{Hz}$, and 


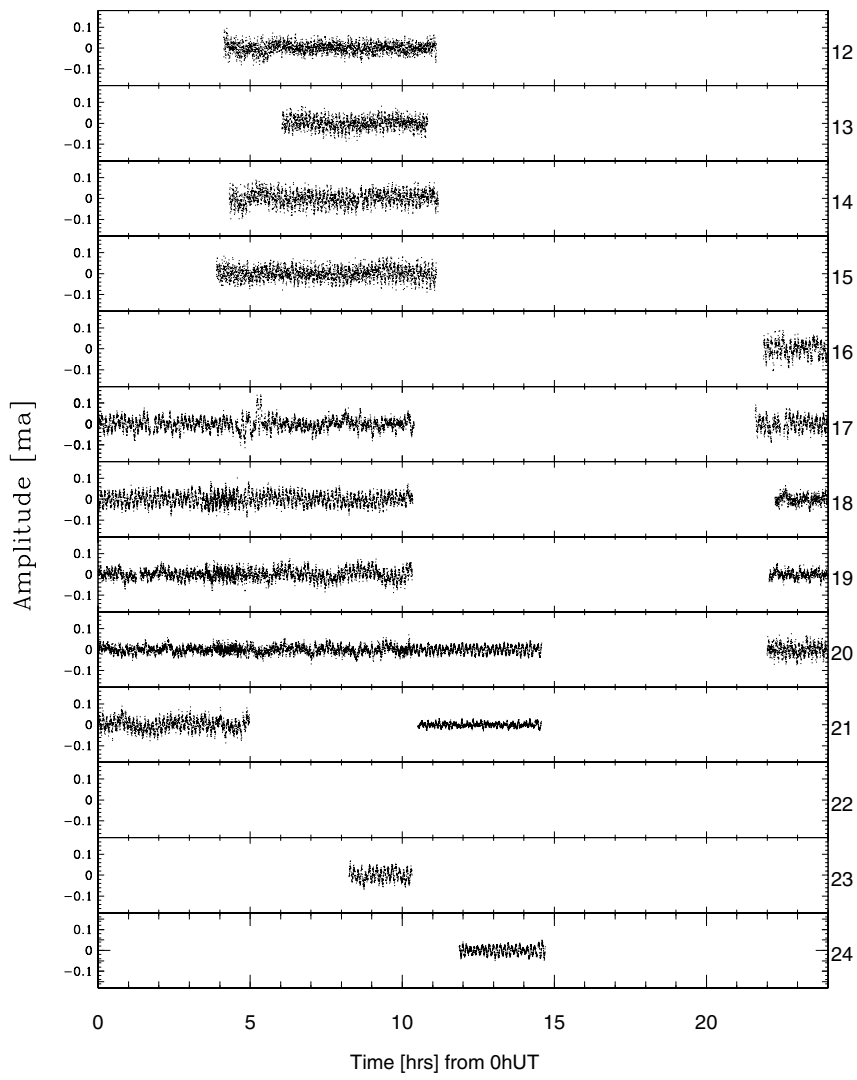

Fig. 1. Light curve of PG $1707+427$ for the main campaign data in Table 1. Dates on the right side of the panel are in May 1991.

allows us to resolve the structures around $1350 \mu \mathrm{Hz}$. The FT of this subset of the data is shown in the left-hand column of Fig. 3. With this core run, we can clearly identify the principal periodicity at $1341 \mu \mathrm{Hz}$; prewhitening reveals a second peak close to $1377 \mu \mathrm{Hz}$.

This figure also shows this region of the FT of the entire WET data set before, during, and after prewhitening by the selected peaks, along with simulations of the region (using the same procedure as we used to produce the window function in Fig. 2 but with multiple periodicities). Again, after removal of the largest peak at $1340.8 \mu \mathrm{Hz}$, the next-highest peak in the region is the $1377.3 \mu \mathrm{Hz}$ peak. Removal of that second peak leaves additional peaks at levels that are consistent with noise in this region.

Table 2 lists the frequencies, amplitudes, and phases of the main peaks seen in the FT of the WET data on PG 1707+427. The column labeled "Rank" relates to the relative amplitudes of the peaks. For all peaks listed in Table 2, the values of the parameters were determined using a simultaneous nonlinear least-squares fit to the lightcurve, with three parameters for each periodicity (frequency, amplitude, and time of first maximum or, equivalently, phase). The uncertainties listed in the table are the formal least-squares estimates of the errors; in practice, the true uncertainty $(1 \sigma)$ is probably somewhat larger.

In Table 2, we identify 7 independent pulsations (ranks 1-7). In addition to these, there are two peaks near $4465 \mu \mathrm{Hz}$. These peaks result from nonlinearities associated with the two largest-amplitude peaks. The first harmonic of the $2236.2 \mu \mathrm{Hz}$ mode appears at $4472.5 \mu \mathrm{Hz}$, and the sum of the frequencies of the $2236.2 \mu \mathrm{Hz}$ and $2227.4 \mu \mathrm{Hz}$ modes appears at $4463.5 \mu \mathrm{Hz}$. These combination frequencies are exact, within the statistical uncertainties of the frequency determinations. Peaks ranked $8-10$ are discussed in the next section.

\subsection{Low amplitude modes recovered with CFHT}

After prewhitening by the seven primary frequencies, the FT of the core WET data still shows several peaks that appear to be higher than the nearby noise levels. Figure 4 shows this prewhitening process. The top panel is the FT of the WET data with the positions of the seven primary frequencies marked. The middle panel shows the FT after removal of those seven peaks, with the next three largest peaks with frequencies above $1000 \mu \mathrm{Hz}$ marked. A least-squares fit easily determines their amplitudes, frequencies, and phases, allowing their removal as shown in the bottom panel of Fig. 4. The result of the least-squares fit is given in Table 2 by the lines marked with asterisks in the Rank column. The noise level in this region of the FT lies at about 1 mma.

Are these also real periodicities in the star, or are they caused by noise?

To answer this question, we appeal to data from the largest telescope in this network, the $3.5 \mathrm{~m} \mathrm{CFH} \mathrm{Telescope.} \mathrm{Data} \mathrm{from}$ the CFHT had the highest signal-to-noise. Therefore, we could expect that lower amplitude modes would be more clearly discerned in FTs of the CFHT data alone. Unfortunately, we only had three relatively short runs from CFHT available, as given in Table 1. Two of these were on consecutive nights (cfh-002 and cfh-003) while the third was obtained three nights later. Therefore, since the window function of the entire CFHT data set would be extremely complex, we chose to analyze the first two, contiguous, nights with their simpler window function.

Indeed the FT of those two runs, as shown in the top panel of Fig. 5, shows a noise level that is about a factor of two smaller than for the whole WET data set. The disadvantage is that the single-site data show large 1 cycle/day aliases. However, for the seven largest modes, we can appeal to the results shown in Table 2 to identify the correct peaks in the CFHT subset. Those peaks are indicated in the top panel of Fig. 5. Using Table 2 as our guide, we prewhiten the CFHT subset by the top seven frequencies in Table 2 (with phases and amplitudes determined using just the CFHT data) to produce the middle panel of Fig. 5.

The middle panel clearly shows several peaks that are well above the noise level. Comparison with Fig. 4 shows that the three peaks marked in that figure are also present in the CFHT subset. The window function of the WET data is much cleaner than for the CFHT subset. Therefore, we selected the proper peak from the alias pattern and performed a least-squares fit to the CFHT data including those three peaks, the seven original peaks, and a peak seen only in the CFHT data at $1040 \mu \mathrm{Hz}$. Removal of those four peaks produces a prewhitened FT that is shown as the bottom panel of Fig. 5. The remaining features could be noise or might represent even smaller-amplitude pulsations of PG $1707+427$. 

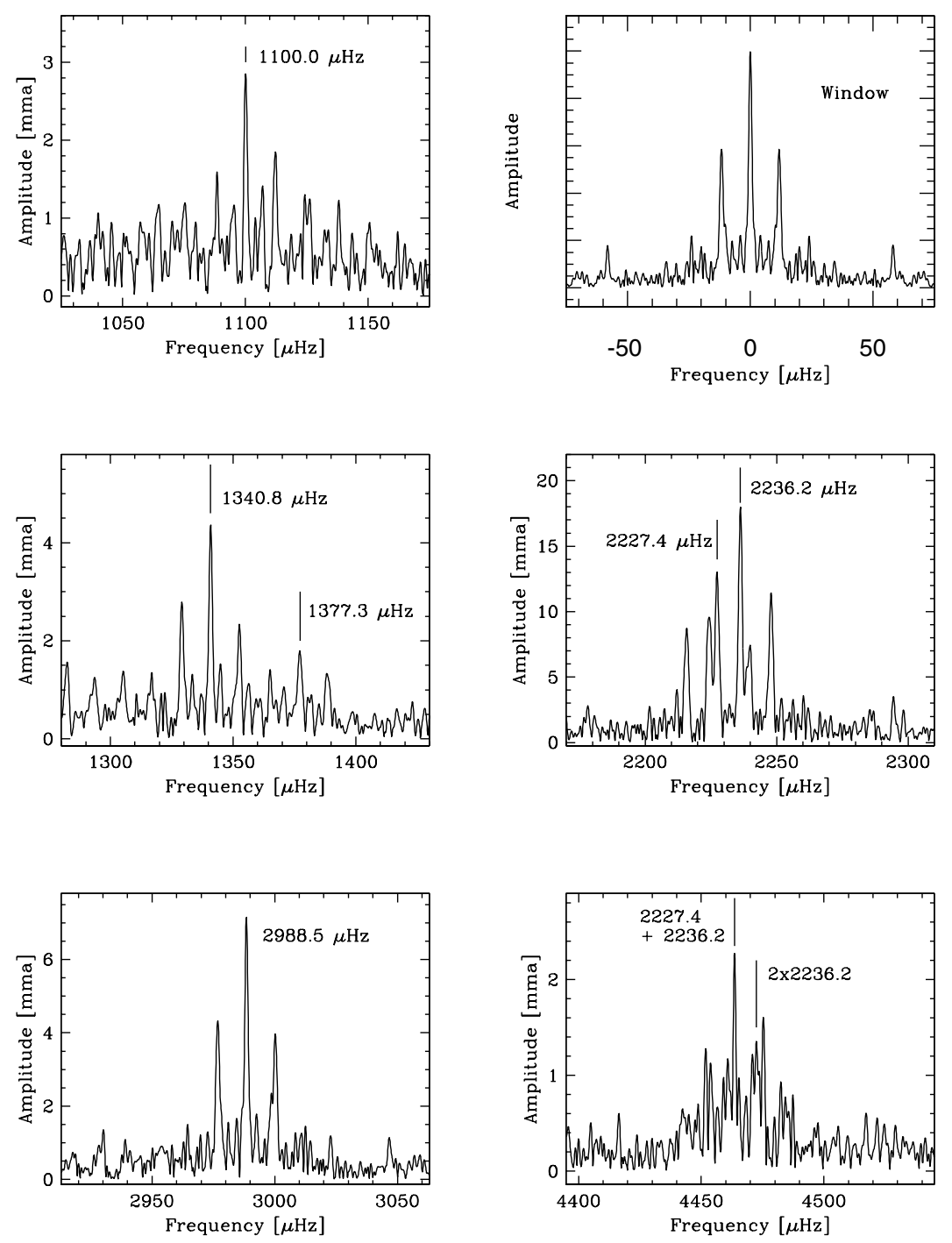

Fig. 2. The main features of the Fourier transform of PG $1707+427$ for the main campaign data in Table 1 - that is, the data from May 1991. Also shown is the Fourier transform of a single-frequency sinusoid to illustrate the spectral window for these data. Obvious (and some not-so-obvious) real frequencies are indicated in each panel.

We note that at all stages in the analysis of the CFHT data, the $1 \mathrm{c} / \mathrm{d}$ window pattern obscures the true peaks. Examination of the top panel of Fig. 5 shows this for the peaks near 1100, 1340, and $2417 \mu \mathrm{Hz}$. The central panels of Figs. 4 and 5 are compared in Fig. 6 - again revealing that the single site data suffer from severe $1 \mathrm{c} / \mathrm{d}$ aliasing, despite their much higher signal-to-noise.

Finally, we compare the results of a nonlinear least-squares fit to these 12-13 frequencies using the WET data (Table 2) and, separately, the data from the CFHT subset (Table 3). The three modes revealed here, at 1475, 1864, and $2023 \mu \mathrm{Hz}$, have the same frequencies (within the uncertainties) in the two data sets. We further note that redoing Fig. 4 excluding the CFHT data produces results that are nearly identical to that shown in the figure. We are therefore convinced that the three lowamplitude modes indicated in Fig. 6 are indeed periodicities in the star.

Curiously, there is a peak present in the CFHT data at $1039.90 \mu \mathrm{Hz}$ that is not present in any of the other data from other sites. This peak is particularly puzzling in that it has an amplitude of $2.6 \mathrm{mmag}$ within the CFHT data and should have shown up in data from other sites. However, we only find this peak in the data from CFHT; it is present within all three individual runs from that telescope. It is not present in the complete data set, however, and so we cannot be sure if that is the correct frequency, or if it is off by a cycle/day or more.

This exercise reveals low-amplitude modes with secure identifications distinguishing them from aliases. This has been achieved by taking advantage of the relatively clean window function of the WET data (despite lower signal-to-noise) combined with the high signal-to-noise identification of peaks in large telescope data (despite the poor FT window). We could not have achieved this without having a large telescope in combination with supporting data from smaller telescopes in a networked observation. Using the single site, large telescope data alone resulted in detection of low-amplitude modes, but the highest peaks within the alias pattern of those modes were never the true periodicities. Identification of the correct periodicity within the alias pattern required supporting data from telescopes at other longitudes.

We note that given the large difference in collecting area (and therefore signal-to-noise) between the telescopes involved in this run, that the CFHT data should carry significantly higher weight. We explored using weights in the computation 


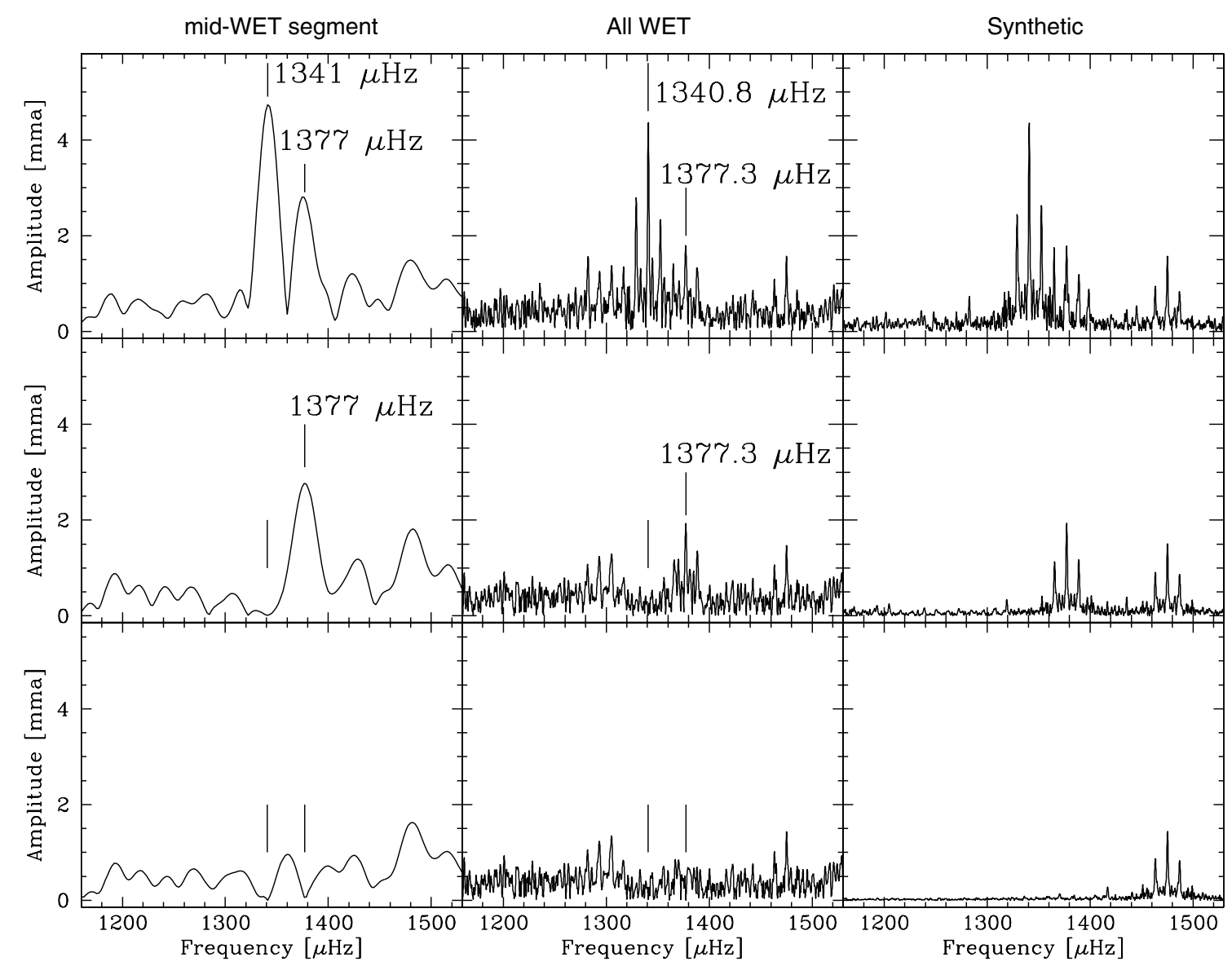

Fig. 3. Enlargement of the region of the FT surrounding the peaks at 1341 and $1377 \mu \mathrm{Hz}$. The panel on the left shows the FT of the longest contiguous stretch of WET data (approximately $18 \mathrm{~h}$ ); the central panel shows the FT of the entire WET data set; and, for comparison, simulated data using the frequencies, amplitudes, and phases in Table 1 to simulate noise-free data with the same time sampling. The top panels show the FT; the center panels show the FT after the $1341 \mu \mathrm{Hz}$ peak has been removed, and the bottom panels show the FT after removing the peak at $1341 \mu \mathrm{Hz}$ and $1377 \mu \mathrm{Hz}$. This represents our simplest solution for this region of the FT; other choices of pairs of peaks let to much less satisfactory matches with synthetic data.

Table 2. Independent pulsation frequencies identified in the combined WET data set. Formal $1 \sigma$ least squares errors are quoted. Peaks with ranks containing $(*)$ are discussed in the text.

\begin{tabular}{ccccc}
\hline \hline $\begin{array}{c}\text { Frequency } \\
{[\mu \mathrm{Hz}]}\end{array}$ & $\begin{array}{c}\text { Period } \\
{[\mathrm{s}]}\end{array}$ & $\begin{array}{c}\text { Amplitude } \\
{[\mathrm{mma}] \pm 0.16}\end{array}$ & $\begin{array}{c}T_{\max } \\
{[\mathrm{s}]}\end{array}$ & Rank \\
\hline $1100.085 \pm 0.040$ & $909.029 \pm 0.033$ & 2.81 & $816 \pm 21$ & 6 \\
$1340.834 \pm 0.026$ & $745.815 \pm 0.014$ & 4.43 & $327 \pm 11$ & 4 \\
$1377.262 \pm 0.060$ & $726.044 \pm 0.031$ & 1.90 & $377 \pm 25$ & 7 \\
$1475.181 \pm 0.078$ & $677.883 \pm 0.036$ & 1.45 & $315 \pm 31$ & $10(*)$ \\
$1864.207 \pm 0.078$ & $536.421 \pm 0.022$ & 1.46 & $455 \pm 24$ & $9(*)$ \\
$2022.590 \pm 0.073$ & $494.416 \pm 0.018$ & 1.55 & $324 \pm 21$ & $8(*)$ \\
$2227.383 \pm 0.010$ & $448.957 \pm 0.002$ & 11.74 & $436 \pm 3$ & 2 \\
$2236.219 \pm 0.007$ & $447.183 \pm 0.001$ & 17.17 & $101 \pm 2$ & 1 \\
$2416.972 \pm 0.036$ & $413.741 \pm 0.006$ & 3.14 & $383 \pm 9$ & 5 \\
$2988.451 \pm 0.016$ & $334.622 \pm 0.002$ & 7.28 & $87 \pm 3$ & 3 \\
$4463.536 \pm 0.045$ & $224.038 \pm 0.002$ & 2.54 & $149 \pm 6$ & $\left(f_{1}+f_{2}\right)-0.043$ \\
$4472.502 \pm 0.076$ & $223.589 \pm 0.004$ & 1.56 & $127 \pm 10$ & $\left(2 \times f_{1}\right)+0.08$ \\
\hline
\end{tabular}




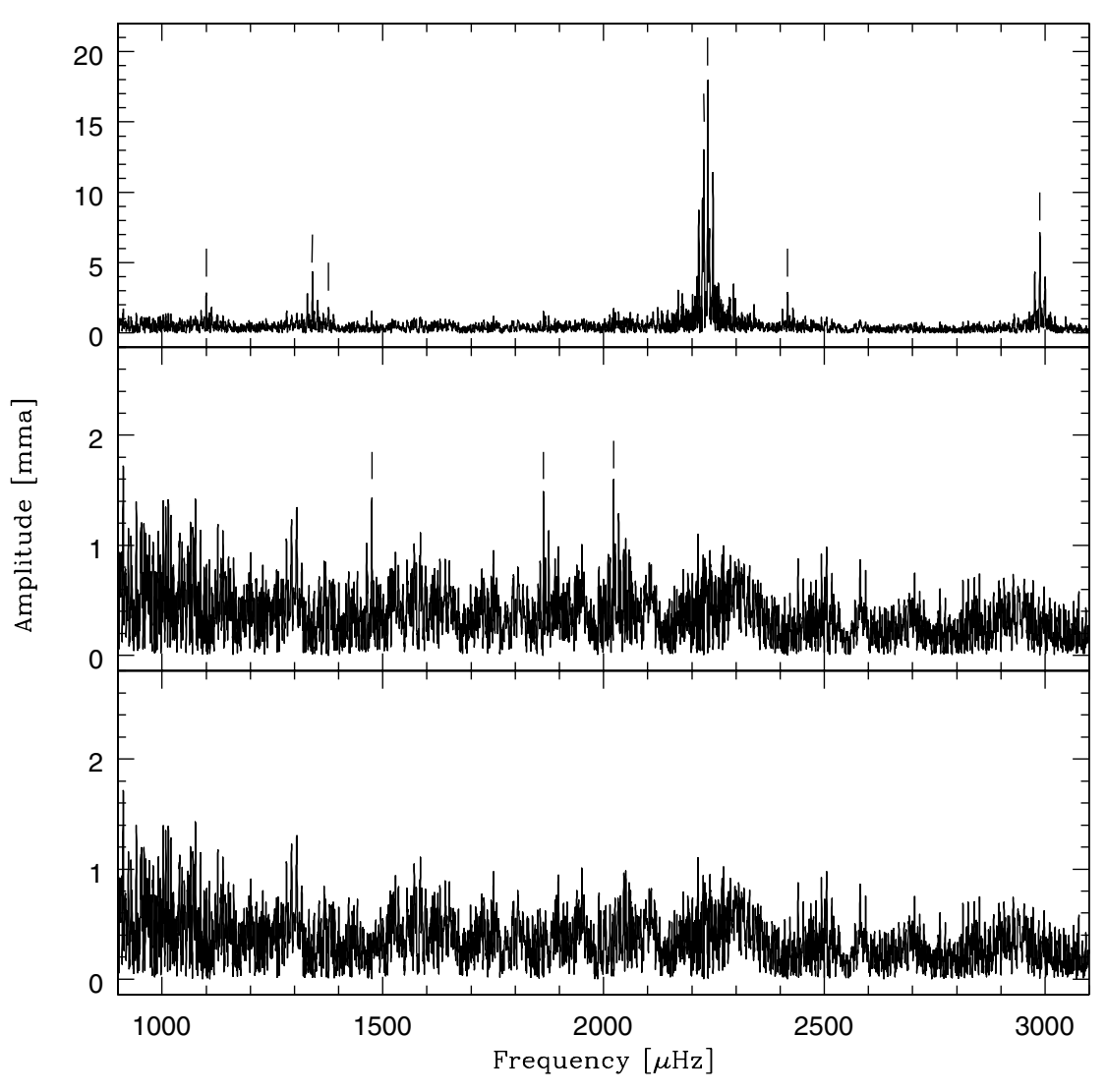

Fig. 4. Fourier transform of the central part of the WET data. The top panel shows the FT of the original data, with peaks identified by vertical lines. In the middle panel, we have prewhitened the data by the indicated peaks (in the time domain), and show the FT of the residuals. Three additional peaks are evident - and appear at much higher signal-to-noise in Fig. 5. The bottom panel shows the FT of the data with all 10 peaks removed. of the FTs of the entire data set to try to reduce the overall noise in the FT while retaining the advantage of multiple longitude coverage (Dind et al. 2003). Results of that exercise revealed the same frequencies as we find using the procedures described in this subsection; this is in accord with the findings of Handler (2003) about use of large and small telescopes together within a WET-style campaign.

Finally, we used the frequency identifications obtained above to do a least-squares fit to all of the data obtained during the 1991 campaign (i.e. all runs in Table 1). With the three runs obtained in June 1991, the frequency precision of the identified modes was greatly improved. The results of this final fit are shown in Table 4.

\section{Analysis of the pulsation spectrum of PG $1707+427$}

\subsection{The strange case of the $9 \mu \mathrm{Hz}$ split of the main mode}

The periods we see in PG $1707+427$ are typical of the naked GW Vir stars; the star shows roughly the same number of peaks as seen in the other naked GW Vir stars. One point, though, is that the range of periods is larger than average, with periods ranging from below $400 \mathrm{~s}$ to over $900 \mathrm{~s}$; we will address the implications of this in a later section.

PG $1707+427$ is dominated by two large-amplitude modes with periods of 447.2 and $449.0 \mathrm{~s}$. These are too close in period to be consecutive radial overtones of the same $\ell$ - typical period spacings seen in the other GW Vir stars are about $21 \mathrm{~s}$ for $\ell=1$ modes and $12 \mathrm{~s}$ for $\ell=2$ modes. A more natural assumption is that these two modes are modes with the same value of $n$ and $\ell$, but different $m$ - that is, they are two modes of a rotationally split multiplet. Their frequency difference is $8.86 \mu \mathrm{Hz}$, which corresponds to a rotation period of 0.65 days (for $\delta m=1$ ) or 1.30 days (for $\delta m=2$ ) if $\ell=1$. These rotation periods are within the range seen for other GW Vir stars that show rotational splittings (i.e. PG 2131+066, NGC 1501, PG 1159-035, and RX J2117+3412).

However, unlike most other GW Vir stars, this splitting is seen only once over the entire frequency range. No other modes show any splitting of this size. Furthermore, while other GW Vir stars show rotationally split triplets (and PG 1159-035 itself shows several $\ell=2$ quintuplets), PG $1707+427$ shows only this pair of modes - a doublet. Despite our best attempts at analysis of the highest signal-to-noise data within our runs, we found no evidence for a third peak in the range from $440 \mathrm{~s}$ to $455 \mathrm{~s}$ that could complete the triplet or indicate a quintuplet. We have no ready explanation for this singular behavior, and the possibility remains that the two peaks are not components of a rotational multiplet with the same $\ell$ and $n$.

Further adding to the story is the pair of peaks near the harmonic of the dominant mode. As we show in the frequency tables, within the least-squares uncertainties, the harmonic of the dominant mode (the $447.2 \mathrm{~s}$ peak) is present in the data. Nearby, but at lower frequency, is another peak with a frequency that is the exact sum of the frequencies of the $447.2 \mathrm{~s}$ mode and the $449.0 \mathrm{~s}$ peak. No other nonlinear combination peaks are present in the Fourier transforms. 


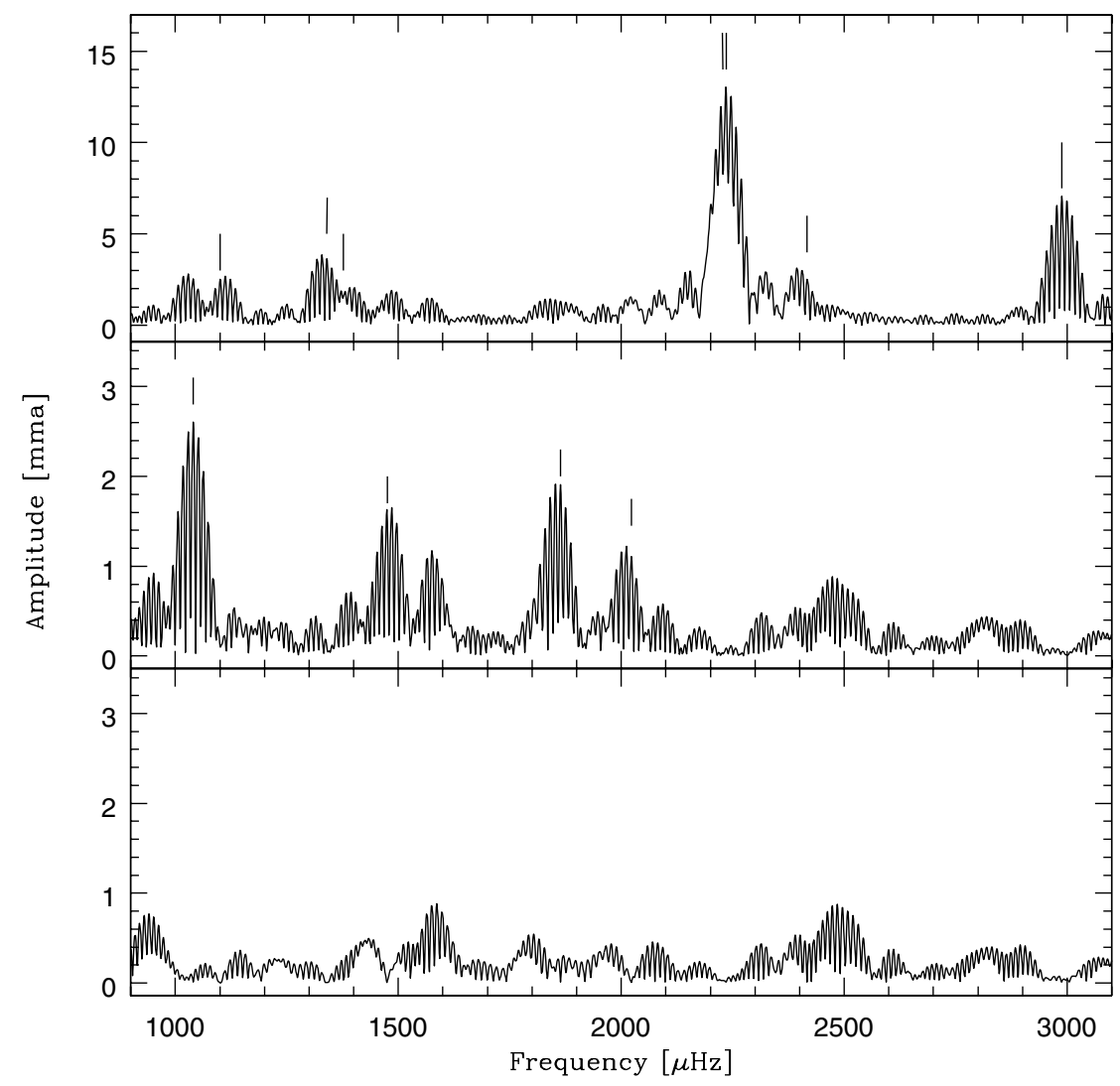

Fig. 5. As in Fig. 4, but Fourier transform of runs cfh-002 and cfh-003, the two highest $\mathrm{S} / \mathrm{N}$ runs during the WET campaign. Given the much better window function of the collected WET data, we can identify the proper peaks from the forest of $1 \mathrm{c} / \mathrm{d}$ aliases in this data set. Three of the four additional peaks are evident - and appear at much higher signal-to-noise in Fig. 4. The bottom panel shows the FT of the data with all 10 peaks removed.

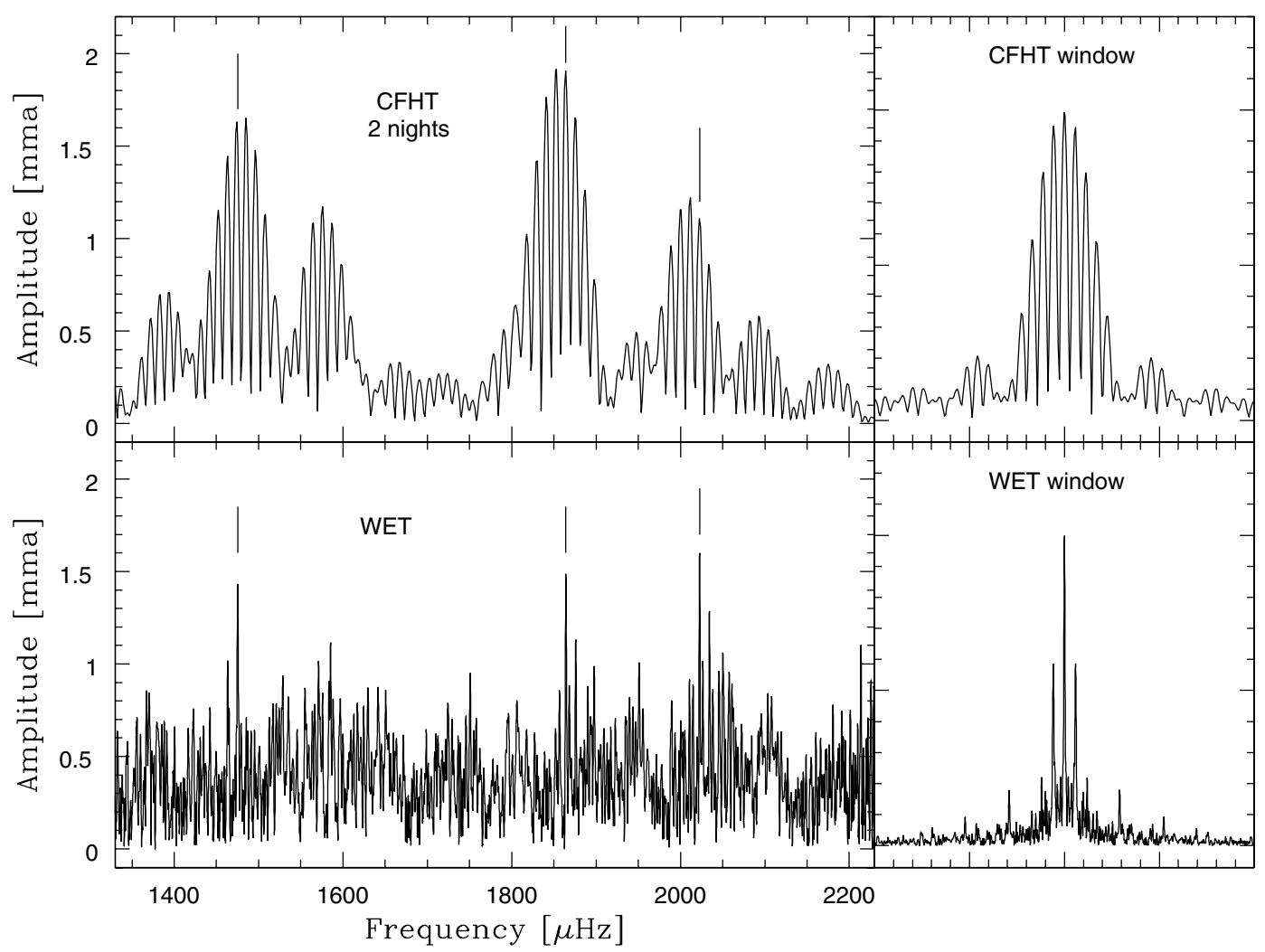

Fig. 6. Comparison of a segment of the FT of PG $1707+427$ using data from the two highest $\mathrm{S} / \mathrm{N}$ runs (cfh-002 and cfh- 003 , top) and the core of the WET data. The associated windows are shown (on the same frequency scale in the right panels. The vertical lines show the frequencies seen in the WET data, repeated in both panels. While the $\mathrm{S} / \mathrm{N}$ of the CFHT data is much better than the WET as a whole, the $1 \mathrm{c} / \mathrm{d}$ aliases mask the real frequencies. On the other hand, the lower S/N WET data show clean FT peaks with minimal alias confusion. Thus we can use the high S/N CFHT data to confirm the reality of individual low-amplitude modes, and definitively break the $1 \mathrm{c} / \mathrm{d}$ aliases by consultation with the WET data. 
Table 3. Pulsation frequencies identified in the combined data from cfh- 002 and cfh- 003 , with aliases broken using the whole WET campaign when available. Formal $1 \sigma$ least squares errors are quoted.

\begin{tabular}{ccccc}
\hline \hline $\begin{array}{c}\text { Frequency } \\
{[\mu \mathrm{Hz}]}\end{array}$ & $\begin{array}{c}\text { Period } \\
{[\mathrm{s}]}\end{array}$ & $\begin{array}{c}\text { Amplitude } \\
{[\mathrm{mma}]}\end{array}$ & $\begin{array}{c}T_{\max } \\
{[\mathrm{s}]}\end{array}$ & Rank \\
\hline $1039.90 \pm 0.49$ & $961.63 \pm 0.46$ & $2.61 \pm 0.35$ & $598 \pm 31$ & \\
$1100.47 \pm 0.54$ & $908.71 \pm 0.45$ & $2.42 \pm 0.36$ & $371 \pm 32$ & 6 \\
$1340.87 \pm 0.33$ & $745.78 \pm 0.18$ & $4.49 \pm 0.43$ & $366 \pm 16$ & 4 \\
$1377.82 \pm 0.58$ & $725.78 \pm 0.30$ & $2.63 \pm 0.36$ & $253 \pm 27$ & 7 \\
$1474.03 \pm 0.82$ & $678.41 \pm 0.38$ & $1.62 \pm 0.36$ & $486 \pm 37$ & \\
$1864.06 \pm 0.72$ & $536.46 \pm 0.21$ & $1.91 \pm 0.35$ & $306 \pm 25$ & \\
$2022.40 \pm 1.30$ & $494.45 \pm 0.32$ & $0.99 \pm 0.36$ & $180 \pm 43$ & \\
$2227.59 \pm 0.39$ & $448.92 \pm 0.08$ & $12.65 \pm 1.02$ & $122 \pm 7$ & 2 \\
$2236.25 \pm 0.28$ & $447.18 \pm 0.06$ & $17.41 \pm 1.02$ & $125 \pm 5$ & 5 \\
$2417.00 \pm 0.52$ & $413.74 \pm 0.09$ & $2.49 \pm 0.36$ & $395 \pm 14$ & 3 \\
$2988.46 \pm 0.18$ & $334.62 \pm 0.02$ & $7.29 \pm 0.43$ & $93 \pm 4$ & \\
& & & & \\
$4464.3 \pm 1.9$ & $224.00 \pm 0.09$ & $2.28 \pm 0.36$ & $165 \pm 20$ & $\left(f_{1}+f_{2}\right)+0.46$ \\
$4472.5 \pm 2.1$ & $223.59 \pm 0.11$ & $2.08 \pm 0.36$ & $125 \pm 10$ & $\left(2 \times f_{1}\right)$ \\
\hline
\end{tabular}

Table 4. Pulsation frequencies identified in all data from the WET run described in the text, including data from three runs one month later. Formal $1 \sigma$ least squares errors are quoted.

\begin{tabular}{ccccc}
\hline \hline $\begin{array}{c}\text { Frequency } \\
{[\mu \mathrm{Hz}]}\end{array}$ & $\begin{array}{c}\text { Period } \\
{[\mathrm{s}]}\end{array}$ & $\begin{array}{c}\text { Amplitude } \\
{[\mathrm{mma}] \pm 0.15}\end{array}$ & $\begin{array}{c}T_{\max } \\
{[\mathrm{s}]}\end{array}$ & Rank \\
\hline $1100.045 \pm 0.008$ & $909.053 \pm 0.006$ & 3.05 & $771 \pm 10$ & 6 \\
$1340.877 \pm 0.005$ & $745.781 \pm 0.003$ & 4.44 & $367 \pm 6$ & 4 \\
$1377.374 \pm 0.012$ & $726.019 \pm 0.006$ & 2.00 & $253 \pm 12$ & 7 \\
$1475.157 \pm 0.018$ & $677.894 \pm 0.008$ & 1.31 & $295 \pm 17$ & \\
$1864.250 \pm 0.017$ & $536.409 \pm 0.005$ & 1.42 & $484 \pm 13$ & \\
$2022.704 \pm 0.012$ & $494.388 \pm 0.003$ & 1.89 & $394 \pm 9$ & \\
$2227.373 \pm 0.002$ & $448.9593 \pm 0.0004$ & 11.46 & $430 \pm 1$ & 2 \\
$2236.232 \pm 0.001$ & $447.1808 \pm 0.0002$ & 16.91 & $109 \pm 1$ & 5 \\
$2416.955 \pm 0.007$ & $413.744 \pm 0.001$ & 3.26 & $374 \pm 4$ & 3 \\
$2988.451 \pm 0.003$ & $334.6215 \pm 0.0004$ & 7.17 & $87 \pm 2$ & \\
$4463.601 \pm 0.009$ & $224.0344 \pm 0.0005$ & 2.54 & $166 \pm 3$ & $\left(f_{1}+f_{2}\right)-0.004$ \\
$4472.477 \pm 0.016$ & $223.5898 \pm 0.0008$ & 1.47 & $119 \pm 5$ & $\left(2 \times f_{1}\right)+0.013$ \\
\hline
\end{tabular}

\subsection{Period spacings in $P G 1707+427$}

Assuming that the two main periodicities share the same $n$ and $\ell$, and the highest frequency peaks are nonlinear combination frequencies, we looked at all of the remaining independent periodicities to try to find the systematics expected of $g$-modes. In particular, we searched for a minimum period difference, as for high order $(n>\ell) g$-modes, modes should be approximately equally spaced in period with increasing $n$. Even if some of the modes are missing, the sequence can still be identified by searching for integral multiples of a common period spacing.
Two methods are commonly used to do this search - both involve assuming the existence of a relation of the form:

$P_{n, \ell}=\frac{\Pi_{\mathrm{o}}}{\sqrt{\ell(\ell+1)}} \times(n+$ constant $)$

where $\Pi_{0}$ is a constant that depends on the structure of the star. The first method employs a Komogorov-Smirnov (K-S) test of the likelihood that the periods are drawn from a distribution that follows that rule above (i.e. Kawaler 1988). The second looks at the inverse variance (IV) of the data from such a model as a function of the $\Pi_{\mathrm{o}}$ (O’Donoghue 1994). 
Table 5. Observed periods compared with an asymptotic model with fixed period spacing of $23.008 \mathrm{~s}$.

\begin{tabular}{cccccc}
\hline \hline$\Delta n$ & $P_{\text {model }}$ & $P_{\text {star }}$ & $P_{\text {star }}-P_{\text {model }}$ & $\Delta P / 23.008$ & Comments \\
\hline 0 & 332.9 & 334.6 & 1.7 & 0.07 & \\
3 & 401.9 & $403.2:$ & 1.3 & 0.06 & residual peak in CFHT data \\
$(3)$ & 401.9 & 413.7 & 11.8 & 0.51 & $\ell=2 ?$ \\
5 & 448.0 & 447.2 & -0.8 & -0.03 & one component of doublet \\
5 & 448.0 & 449.0 & 1.0 & 0.04 & one component of doublet \\
7 & 494.0 & 494.4 & 0.4 & 0.02 & \\
9 & 540.0 & 536.4 & -3.6 & -0.16 & \\
13 & 632.0 & $632.5:$ & 0.5 & 0.02 & residual peak in CFHT data \\
15 & 678.0 & 677.9 & -0.1 & -0.004 & \\
17 & 724.0 & 726.0 & 2.0 & 0.09 & \\
18 & 747.0 & 745.8 & -1.2 & -0.05 & \\
25 & 908.1 & 909.0 & 0.9 & 0.04 & \\
\hline
\end{tabular}

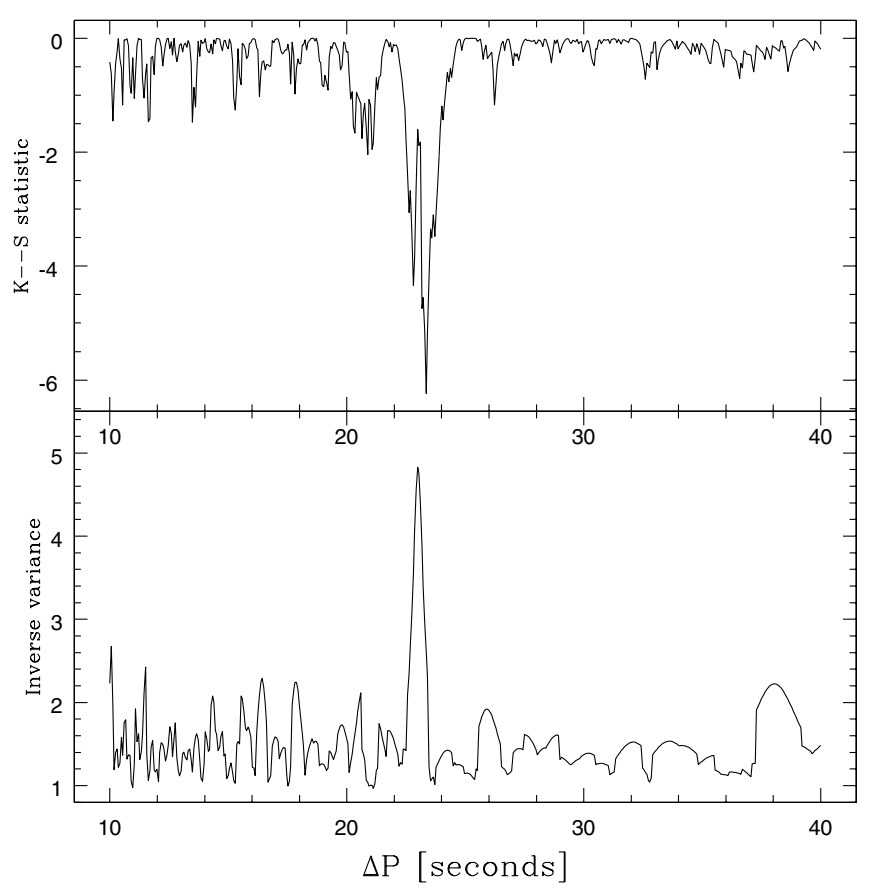

Fig. 7. Two statistical tests of the significance of the common period spacing seen in the periods of PG $1707+427$. For both tests, we used all of the independent periods in Table 4 except for the $413.7 \mathrm{~s}$ period. The top panel shows the results of a Kolmogorov-Smirnov test, and the bottom panel shows the inverse variance of the periods compared to a constant period spacing model.

Using all of the independent periods in Table 4, both the $\mathrm{K}-\mathrm{S}$ test and the IV test show a weak indication of a period spacing of $23.3 \mathrm{~s}$. However, we also found that one of the periods in Table 4 was responsible for most of the $\chi^{2}$ of the fit: the period at $413.7 \mathrm{~s}$. Excluding just that period from the $\mathrm{K}-\mathrm{S}$ and IV tests resulted in a very significant "signal" at $23.0 \mathrm{~s}$ for the mean period spacing. Figure 7 shows the results of these two tests using all of the periods except the $413.7 \mathrm{~s}$ period.

The best value for the period spacing was determined by a least-squares regression to the above equation using the set of periods indicated in Fig. 7. This yielded a value of $\Pi_{\mathrm{o}}=$ $23.008 \pm 0.002 \mathrm{~s}$ and a constant of $332.9 \pm 0.4 \mathrm{~s}$. In Table 5, we list the observed periods and compare them with the model above and a fixed period spacing of 23.008 s. Clearly, each mode (except for the $413.7 \mathrm{~s}$ mode) is comfortably close to that predicted by this simple model. We see the largest departure in the mode with a period of $536.4 \mathrm{~s}$, which is still within $4 \mathrm{~s}$ of the model. In more realistic simulations, the period spacings can vary by a significant fraction of the mean period spacing as the result of resonant mode trapping. With this in mind, the concordance between the observed periods and those of a constant spacing model is very strong.

In addition to the periods from our data that we consider well-determined, two additional periods are included in Table 5. These are the two highest remaining peaks on the power spectrum between 1500 and $3000 \mu \mathrm{Hz}$ in the high signalto-noise data (from the CFHT) in the bottom panel of Fig. 5. The two regions are near $1580 \mu \mathrm{Hz}$ and $2500 \mu \mathrm{Hz}$. We picked the highest peak within the forest of aliases in each thicket to obtain the periods used in the table. In both cases, these low-amplitude modes fit the predictions of the constant period spacing model to a remarkable degree, and we include them in Table 5. While our identification of true periodicities in bands could be off by a cycle/day, the period difference between aliases in these two groups is at most $2 \mathrm{~s}$ for the peak near $403 \mathrm{~s}$, and $5 \mathrm{~s}$ for the peak near $632 \mathrm{~s}$. Therefore we are optimistic that these two modes are real as well, and could be used in seismic modeling.

We note that the failure of finding a fit to the above period spacing model when including the $413.7 \mathrm{~s}$ mode suggests that it has a different value of $\ell$ from the other modes. Given the broad mass constraints on $\Pi_{0}$, the $23 \mathrm{~s}$ mean period spacing requires $\ell=1$. Therefore, it is likely that the $413.7 \mathrm{~s}$ mode is $\ell=2$ (or perhaps even higher). The fact that the period difference between it and the nearest secure mode is nearly 1.5 times the mean period spacing might suggest that the overall period spacing for the star is $11.5 \mathrm{~s}$ (marginally consistent with all modes being $\ell=2$ ) but this is not supported by the KolmogorovSmirnov or inverse variance tests shown in Fig. 7. 
Another mode that turned up in our analysis, the $1039.90 \mu \mathrm{Hz}$ peak seen in the CFHT data, has a period of $961.6 \mathrm{~s}$. At this low frequency, the 1 cycle/day alias uncertainty corresponds to a period change of $10.6 \mathrm{~s}$, and given the fact that this mode appears only in the CFHT data, the period could therefore be $10.6 \mathrm{~s}$ higher or lower as well (i.e. $951.0 \mathrm{~s}$ or $972.4 \mathrm{~s}$ ). The nearest periods expected from a constant period spacing model are at $953.8 \mathrm{~s}$ and $976.7 \mathrm{~s}$. So, if the true frequency is 1 cycle/day higher than the peak in the CFHT data, the model value lies within $2.8 \mathrm{~s}$. Alternatively, the frequency difference between a mode at $953.8 \mathrm{~s}$ and the observed period of $961.6 \mathrm{~s}$ is $8.5 \mu \mathrm{Hz}$ - which very nearly the same as the spitting of the dominant periodicites of $2227.4 \mu \mathrm{Hz}$ and $2236.2 \mu \mathrm{Hz}$. Since this mode was seen only from one site, and given the uncertainties inherent in the above stretch to fit the model, we make no further claims about that mode.

Finally, we note that Fontaine et al. (1991) found a different period spacing for PG $1707+427$ based on a single night of high signal-to-noise data from CFHT. The value they obtained was $18.37 \mathrm{~s}$, using 8 peaks in their data (including the discrepant mode near $413 \mathrm{~s}$ ). Comparing our data (with a much higher frequency resolution) with the earlier result, we find that the five shortest period modes in Fontaine et al. (1991) are essentially the same as what we have found. However, their three longest period modes differ considerably from what we find. This is simply the result of the limited frequency resolution of a single night's run. Fontaine et al. (1991) used a single $4 \mathrm{~h}$ run; thus the peaks in the power spectrum have a width of about $70 \mu \mathrm{Hz}$. In the $650-920 \mathrm{~s}$ period range, we now know that at least four modes are present, with two of the frequency spacings of $37 \mu \mathrm{Hz}$ and $100 \mu \mathrm{Hz}$. Even least-squares fitting of individual peaks would only improve this accuracy to a precision of about $7 \mu \mathrm{Hz}(=0.1 / 4 \mathrm{~h})$. This results in an uncertainty in the period of $3.5 \mathrm{~s}$ at $700 \mathrm{~s}$, and $5.6 \mathrm{~s}$ at $900 \mathrm{~s}$. Thus, in hindsight, it is easy to understand why the resulting period list in Fontaine et al. (1991) looks very different than the current list for long-period modes... and why that work suggested a different period spacing.

\subsection{Comparison with standard evolutionary models}

Before considering the asteroseismic constraints, the bulk properties of PG $1707+427$ can be determined solely through spectroscopy. For hot stars like this, however, the constraints on $\log g$ are very weak. Dreizler \& Heber (1998) fit models with only two choices for $\log g$ of 7.0 and 7.5 for such stars, and assign PG $1707+427$ a gravity of 7.5 , with an uncertainty of something less than 0.5 . The effective temperature of $85000 \mathrm{~K}$ has a $10 \%$ uncertainty.

Coupling these to representative evolutionary tracks, Dreizler \& Heber (1998) derive a mass for PG 1707+427 of $0.54 M_{\odot}$. Determining the mass of the star from spectroscopy alone is complicated by the large uncertainty in $\log g$. Simply put, that translates into an uncertainty in $\log \left(L / L_{\odot}\right)$ of 0.5 , or a factor of three, for a fixed mass. The spectroscopic mass determination uses $\log g$ and $T_{\text {eff }}$ to select the mass from white dwarf evolutionary tracks. This large uncertainty in $\log g$ or equivalently $\log \left(L / L_{\odot}\right)$ encompasses evolutionary tracks of masses ranging from below $0.5 M_{\odot}$ to above $0.7 M_{\odot}$ at that effective temperature!

Using our determination of $\Delta \Pi$ of $23.0 \mathrm{~s}$, and realizing that this period spacing depends primarily on the mass of the star (Kawaler \& Bradley 1994), we use Eq. (2) of O'Brien (2000) (which includes a correction to the sign error on the luminosity term in the equation within Kawaler \& Bradley 1994) to make an initial estimate of the mass of PG $1707+427$ of $0.565 M_{\odot}$. Using that mass we then obtain a luminosity of $23 L_{\odot}$ for $\log g=7.5$. Allowing for the spectroscopic uncertainty in $\log \left(L / L_{\odot}\right)$, we then note that even though the mass of a GW Vir model with a given period spacing increases with decreasing luminosity, the luminosity dependence is very weak. Therefore, the uncertainty in the asteroseismic mass for PG $1707+427$ resulting from the possible luminosity range is only that the true mass of PG $1707+427$ could be up to $5 \%$ higher than this value.

\section{Conclusions}

With this paper, we complete an initial survey of the pulsation frequencies in the pulsating PG 1159 stars. PG 1707+427 shows at least seven independent pulsation frequencies. Most of the modes are low-amplitude (less than $3 \mathrm{mma}$ ), and a few are seen only in data of high signal-to-noise. The largest amplitude mode has a companion (the second largest amplitude) only $9 \mu \mathrm{Hz}$ away that could be evidence for rotational splitting. The two highest-frequency modes are linear combinations of those two large-amplitude modes.

All but one of the independent pulsation frequencies are closely fit by a model with a uniform period spacing of $23 \mathrm{~s}$. One mode does not fit this pattern, and could be a mode with $\ell=2$. This uniform period spacing implies an asteroseismic mass of $0.57 M_{\odot}$.

For the four naked stars, the asteroseismic masses that are consistent within the (large) uncertainties of spectroscopic mass. PG $1707+427$ is remarkable, however, in that it is the lowest-mass of the sample, and also shows the largest period spacing. In Table 6, we include our result with others previously quoted by O'Brien (2000) and others.

One of the trends that O'Brien (2000) noted was that, at the time, the mean period spacing was roughly the same for all of the GW Vir stars. He attributed that to a correlation between the luminosity of the star and the mass. That is, the lower luminosity GW Vir stars, which would have longer periods for a given mass (and therefore larger period spacings), actually have somewhat larger masses than their hotter more luminous analogs. Their larger mass therefore reduces the periods, returning the mean period spacing towards the magical 21.5 s. PG $1707+427$, in some sense, confirms this trend. It has a period spacing that is significantly longer than the other GW Vir stars, and a lower luminosity - all of which point towards its having a lower mass than either PG $2131+066$ or PG 0122+200.

Another trend noted by O'Brien (2000) is that pulsators show a "mean period" that decreases with decreasing luminosity. PG 1707+427 follows that trend, but given its lower 
Table 6. Properties of pulsating hot white dwarfs.

\begin{tabular}{cccccccl}
\hline \hline Star & $T_{\text {eff }}[\mathrm{kK}]$ & $\log g$ & $\log \left(L / L_{\odot}\right)$ & $M_{\text {spec }} / M_{\odot}$ & $\Delta \Pi$ & $M_{\text {puls }} / M_{\odot}$ & References \\
\hline NGC 1501 & $135 \pm$ & {$[6.2]$} & {$[3.70]$} & & $22.3 \pm 0.3$ & $0.55 \pm 0.03$ & 1 (spec), 2 (puls) \\
RX J2117+341 & $170 \pm 10$ & $6.0 \pm 0.3$ & $4.05_{-0.32}^{+0.23}$ & $0.7 \pm 0.2(?)$ & $21.48 \pm 0.04$ & $0.56 \pm 0.03$ & 3 (spec),4 (puls) \\
& & & & & & & \\
PG 1159-035 & $140 \pm 6.0$ & $7.0 \pm 0.3$ & $2.7 \pm 0.4$ & $0.54 \pm 0.1$ & $21.5 \pm 0.1$ & $0.59 \pm 0.01$ & $5($ spec), 6 (puls) \\
PG 2131+066 & $95 \pm 5.0$ & $7.5 \pm 0.3$ & $1.6 \pm 0.3$ & $0.55 \pm 0.1$ & $21.6 \pm 0.4$ & $0.61 \pm 0.02$ & $5($ spec), 7 (puls) \\
PG 1707+427 & $85 \pm 4.5$ & $7.5 \pm 0.3$ & $1.4 \pm 0.3$ & $0.54 \pm 0.1$ & $23.0 \pm 0.3$ & $0.57 \pm 0.02$ & $5($ spec), 8 (puls) \\
PG 0122+200 & $80 \pm 4.0$ & $7.5 \pm 0.3$ & $1.3 \pm 0.3$ & $0.53 \pm 0.1$ & $21.1 \pm 0.4$ & $0.69 \pm 0.04$ & $5($ spec), 9 (puls) \\
\hline
\end{tabular}

Reference: 1: Koesterke \& Hamann (1997); 2: Bond et al. (1996); 3: Rauch \& Werner (1997); 4: Vauclair et al. (2002);

5: Dreizler \& Heber (1998); 6: Kawaler \& Bradley (1994); 7: Reed et al. (2000); 8: this work; 9: O’Brien (2000).

mass than PG $0122+200$ (for example) it should not. In trying to identify the factors that determine the instability region along pre-white dwarf evolutionary tracks, O’Brien (2000) suggested a very narrow strip surrounding the locus of places where the period spacing is about $21.5 \mathrm{~s}$. Our finding here is that PG $1707+427$ has a period spacing of $23 \mathrm{~s}$, and therefore extends the "red edge" of O'Brien's locus towards lower $T_{\text {eff }}$.

As O'Brien (2000) states, though, the discovery of a relatively low-mass pulsator on the cool end of the naked GW Vir region implies that longer periods should be present than in, for example, PG $0122+200$ or PG $2131+066$. The longest period seen in PG $0122+200$ is at about $610 \mathrm{~s}$ (O'Brien et al. 1998). PG 2131+066 pulsates with periods only as long as $508 \mathrm{~s}$ (Kawaler et al. 1995). The fact that PG $1707+427$ shows several periods near $750 \mathrm{~s}$, and one at slightly above $900 \mathrm{~s}$, is therefore of interest as well. O'Brien (2000) uses energy arguments from Hansen et al. (1985) to compute the maximum available period as a function of mass and $T_{\text {eff }}$; for $0.57 M_{\odot}$ and $T_{\text {eff }}=85 \mathrm{kK}$, this calculation reveals that the maximum periods are to be expected at about $860 \mathrm{~s}$. This is entirely consistent with what we see in PG $1707+427$.

Hence, our identification of the $23 \mathrm{~s}$ period spacing in PG 1707+427, and therefore our determination of its mass, extends the expected region of instability of the GW Vir stars over what O'Brien (2000) suggested. But, the observed longerperiod modes that the star shows are still consistent with the systematics of the evolution of the driving region in the naked GW Vir stars. With this in mind, several areas of further investigation of the phenomena of hydrogen-poor hot white dwarfs can take advantage of the asteroseismic constraints. First, new models that can explain the systematics of the instability region need to address the trends of periods with luminosity (or effective temperature) and mass. Since we have only used the mean period spacing in this first analysis, a second avenue to explore is more detailed modeling of the interior of the GW Vir stars (including the pulsating central stars of planetary nebulae). The departures from equal period spacing seen in PG $1707+427$ (and the other pulsators) are trying to tell us something about the internal structure of these stars - with the completion of our assay of the class, it is time to listen to the music of asteroseismology to finally decode their origins.
Acknowledgements. S.D.K., E.P., and M.V. were partially funded by NSF grants AST9876655 and AST20205983 and by the NASA Astrophysics Theory Program through grant NAG-58352. P.B., G.F., and F.W. recognize support from the NSERC Canada and FQRNT (Québec). Results based, in part, on observations gathered at the Canada-France-Hawaii Telescope, operated by the National Reserch Council of Canada, the Centre National de la Recherche Sientifiques de France, and the University of Hawaii. P.M. is supported in part by Polish KBN grants 5-P03D-012-020 and 5-P03D-030-20. SJOT was supported by an Australian Postgraduate Award. We also thank Elia Leibowitz and Tsevi Mazeh (Tel Aviv University) for their participation in Xcov6.

\section{References}

Bond, H. E., \& Grauer, A. D. 1984, ApJ, 279, 751

Bond, H. E., Kawaler, S. D., Ciardullo, R., et al. 1996, AJ, 112, 2699

Cox, A. N. 2003, ApJ, 585, 975

De Marco, O. 2002, Ap\&SS, 279, 157

Dind, Z. E., Bedding, T. R., O’Toole, S. J., \& Kawaler, S. D. 2003, in Asteroseismology Across the HR Diagram, ed. M. Cunha, \& M. Thompson (Dordrecht: Kluwer), 417

Dreizler, S., \& Heber, U. 1998, A\&A, 334, 618

Fontaine, G., Bergeron, P., Brassard, P., et al. 1991, ApJ, 378, L49

Górny, S. K., \& Tylenda, R. 2000, A\&A, 362, 1008

Grauer, A. D., Green, R. F., \& Liebert, J. 1992, ApJ, 339, 686

Handler, G. 2003, Balt.Astron., 12, 253

Hansen, C. J., Winget, D. E., \& Kawaler, S. D. 1985, ApJ, 297, 544

Herwig, F. 2001, Ap\&SS, 275, 15

Iben, I. Jr. 1984, ApJ, 277, 333

Iben, I. Jr., \& MacDonald, J. 1995, in White Dwarfs: Proc. of the 9th European White Dwarf Workshop, ed. D. Koester, \& K. Werner (Berlin: Springer), 48

Iben, I. Jr., \& Tutukov, A. V. 1986, ApJ, 311, 753

Iben, I. Jr., Kaler, J., Truran, J., \& Renzini, A. 1983, ApJ, 264, 605

Kawaler, S. D. 1988, in IAU Symp., 123, ed. J. Christensen-Dalsgaard, \& S. Frandsen (Dordrechg: Reidel), 329

Kawaler, S. D., \& Bradley, P. A. 1994, ApJ, 427, 415

Kawaler, S. D., O'Brien, M. S., Clemens, J. C., et al. (the WET Collaboration) 1995, ApJ, 450, 350

Kleinman, S. J., Nather, R. E., \& Phillips, T. 1996, PASP, 108, 356

Koesterke, L., \& Hamann, W.-R. 1997, in Planetary Nebula, ed. H. J. Habing, \& H. J. G. L. M. Lamers (Dordrecht: Kluwer), IAU Symp., 180, 114 
Lawlor, T., \& MacDonald, J. 2003, ApJ, 583, 913

Nather, R. E., Winget, D. E., Clemens, J. C., Hine, B. P., \& Hansen, C. J. 1990, ApJ, 361, 309

O'Brien, M. S. 1998, Ph.D. Thesis, Iowa State University

O'Brien, M. S. 2000, ApJ, 532, 1078

O'Brien, M. S., Vauclair, G., Kawaler, S. D., et al. (the WET collaboration) 1998, ApJ, 495, 458

O’Donoghue, D. O. 1994, MNRAS, 270, 222

Pfeiffer, B., Vauclair, G., Dolez, N., et al. (the WET collaboration) 1996, A\&A, 314, 182

Quirion, P.-O., Fontaine, G., \& Brassard, P. 2004, ApJ, 610, 436

Rauch, T., \& Werner, K. 1997, in 3rd Conference on Faint Blue Stars, ed. A. G. D. Philip, J. Liebert, \& R. A. Saffer (Schenectady: L. Davis Press), 217

Reed, M. D., Kawaler, S. D., \& O’Brien, M. S. 2000, ApJ, 545, 429
Starrfield, S. G., Cox, A. N., Hodson, S. W., \& Pesnell, W. D. 1983, ApJ, 268, L27

Starrfield, S., Cox, A. N., Kidman, R. B., \& Pesnell, W. D. 1984, ApJ, 281,800

Starrfield, S., Cox, A. N., Kidman, R. B., \& Pesnell, W. D. 1985, ApJ, 239, L23

Vauclair, G., et al. (the WET collaboration) 2001, in The 12th European Workshop on White Dwarfs, ed. J. Provencal, H. Shipman, J. MacDonald, \& S. Goodchild (San Francisco: ASP), 293

Vauclair, G., Moskalik, P., Pfeiffer, B., et al. (the WET collaboration) 2002, A\&A, 381, 122

Werner, K. 2001, Ap\&SS, 275, 27

Winget, D. E., Nather, R. E., Clemens, J. C., et al. (the WET collaboration) 1991, ApJ, 378, 326 